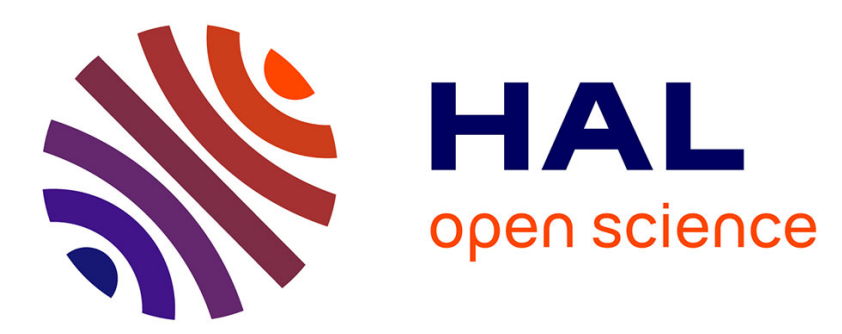

\title{
A Markov chain model of mixing kinetics for ternary mixture of dissimilar particulate solids
}

Vadim Mizonov, Ivan Balagurov, Henri Berthiaux, Cendrine Gatumel

\section{To cite this version:}

Vadim Mizonov, Ivan Balagurov, Henri Berthiaux, Cendrine Gatumel. A Markov chain model of mixing kinetics for ternary mixture of dissimilar particulate solids. Particuology , 2017, 31, p. 80-86. 10.1016/j.partic.2016.05.006 . hal-01619239

\section{HAL Id: hal-01619239 \\ https://hal.science/hal-01619239}

Submitted on 7 Nov 2019

HAL is a multi-disciplinary open access archive for the deposit and dissemination of scientific research documents, whether they are published or not. The documents may come from teaching and research institutions in France or abroad, or from public or private research centers.
L'archive ouverte pluridisciplinaire HAL, est destinée au dépôt et à la diffusion de documents scientifiques de niveau recherche, publiés ou non, émanant des établissements d'enseignement et de recherche français ou étrangers, des laboratoires publics ou privés. 


\title{
A Markov chain model of mixing kinetics for ternary mixture of dissimilar particulate solids
}

\author{
Vadim Mizonov $^{\mathrm{a}, *}$, Ivan Balagurov ${ }^{\mathrm{a}}$, Henri Berthiaux ${ }^{\mathrm{b}}$, Cendrine Gatumel ${ }^{\mathrm{b}}$ \\ a Department of Applied Mathematics, Ivanovo State Power Engineering University, Russia \\ ${ }^{\mathrm{b}}$ Centre RAPSODEE, UMR CNRS 5302, Ecole des Mines d'Albi-Carmaux, Campus Jarlard, Route de Teillet, 81000 Albi, France
}

\begin{abstract}
A B S T R A C T
This paper presents a simple but informative mathematical model to describe the mixing of three dissimilar components of particulate solids that have the tendency to segregate within one another. A nonlinear Markov chain model is proposed to describe the process. At each time step, the exchange of particulate solids between the cells of the chain is divided into two virtual stages. The first is pure stochastic mixing accompanied by downward segregation. Upon the completion of this stage, some of the cells appear to be overfilled with the mixture, while others appear to have a void space. The second stage is related to upward segregation. Components from the overfilled cells fill the upper cells (those with the void space) according to the proposed algorithm. The degree of non-homogeneity in the mixture (the standard deviation) is calculated at each time step, which allows the mixing kinetics to be described. The optimum mixing time is found to provide the maximum homogeneity in the ternary mixture. However, this "common" time differs from the optimum mixing times for individual components. The model is verified using a lab-scale vibration vessel, and a reasonable correlation between the calculated and experimental data is obtained.
\end{abstract}

\author{
Keywords: \\ Ternary mixture \\ Segregation \\ Mixing kinetics \\ Markov chain \\ Matrix of transition probabilities \\ Optimum mixing time
}

\section{Introduction}

The mixing of powders and granular materials is of central importance for the quality and performance of a wide range of products. Bridgwater $(2010,2012)$ emphasized the difficulty of designing and operating the mixing process, which is largely based on judgment rather than science. The next stage of development is to build on the emerging knowledge and methods so as to clarify the basics for such designs. This will enable the process to be conducted in such a way that the mixing operation can be effectively controlled. One of the key problems in mixing dissimilar granular materials is their segregation into one another. The segregation occurs because of differences in the physical properties of the components, such as particle size, density, and shape. The action of gravity, which is always present in mixing, varies for different sorts of particles, and also leads to their segregation. With no segregation, achieving a homogeneous mixture simply involves determining an adequate mixing time. Very often, it is virtually impossible to

\footnotetext{
* Corresponding author. Fax: +7 4932385701.

E-mail address: mizonov46@mail.ru (V. Mizonov).
}

achieve a homogeneous mixture if segregation occurs. First, the homogeneity of a mixture increases, reaches a maximum, and then decreases again. There have been a number of studies, mostly experimental, on the influence of the segregation effect on mixture quality (e.g., Jha \& Puri, 2010; Jha, Gill, \& Puri, 2008; Tang \& Puri, 2007). However, the effect of segregation on the mixing kinetics has received less attention. In particular, it is important to estimate this effect for the mixing of multi-component dissimilar materials, when segregation becomes very complex. In a binary mixture, one component experiences downward segregation and the other component experiences upward segregation. In a ternary mixture, an intermediate component experiences both downward and upward segregation, and the evolution of its distribution becomes difficult to predict.

One way to understand the process better is to build a suitable mathematical model. Different approaches have been used to model the mixing of solids. Danckwerts (1953) and Sommer (1996) developed models based on the forced diffusion equation. These models played an important role in better understanding the mixing process and estimating the quality of mixtures. However, the analytical solutions obtained by this approach are mainly of purely academic interest. This is because of several unrealistic assump- 


\begin{tabular}{|ll|}
\hline \multicolumn{2}{|c|}{ Nomenclature } \\
$D$ & Dispersion coefficient, $\mathrm{m}^{2} / \mathrm{s}$ \\
$d$ & Probability of pure stochastic (diffusion) transition \\
$j$ & Cell number counted from the top of the mixture \\
$k$ & Transition number \\
$H$ & Mixture height \\
$m$ & Total number of cells in the chain \\
$\mathbf{P}, P_{i j}$ & Matrix of transition probabilities and its entries \\
$\mathbf{S}, S_{j}$ & State vector and its entries \\
$S_{\mathrm{max}}$ & Maximum content of mixture that a cell can contain \\
$t$ & Time, s \\
$V$ & Velocity of segregation, $\mathrm{m} / \mathrm{s}$ \\
$v$ & Probability (rate) of segregation transition \\
& \\
Greek symbols & Standard deviation \\
$\sigma$ & Transition duration, $\mathrm{s}$ \\
$\Delta t$ & Height of a cell, $\mathrm{m}$ \\
\multicolumn{2}{|l}{} \\
Indices & \\
1 & Fine fraction \\
2 & Middle fraction \\
3 & Coarse fraction \\
\hline
\end{tabular}

tions and the neglect of important physical features of the process. Kinetic theory was used by Iddir, Arastoopour, and Hrenya (2005) to model the granular mixture of components with different mechanical properties (size, density, and/or restitution coefficient), where each particle group was considered as a separate phase with different average velocity and granular energy. This model was applied to the simple shear flow of binary and ternary mixtures of particles. However, this model mostly concerned the dynamic properties of already formed mixtures rather than the kinetics of their formation. Bridgwater (2010, 2012) used discrete element methods (DEMs) to solve the main problems of mixing. However, this approach is very time consuming, which becomes a serious problem when calculating and comparing numerous process regimes and mixer configurations.

From the authors' viewpoint, one tool that is capable of solving these problems is the theory of Markov chains, which is related to the process of mixing as it describes the evolution of the state of a stochastic system. The basic idea of the Markov chain approach consists of separating the operating volume of the mixer into small but finite zones (cells) and then observing the evolution of the key component concentration in these zones at discrete moments in time, with a small but finite time step between them. This approach was used by Wang and Fan (1976) to describe the state of a mixture after passing through a static mixer. However, their work neglected the evolution of the process parameters, and did not describe the physical features of the mixing zone. In later studies by these researchers (Fan, Lai, Akao, Shinoda, \& Yoshizawa, 1978; Wang \& Fan, 1977), a model was developed in which transitions were only permitted to the neighboring cells.

Doucet et al. (2008)Doucet, Hudon, Bertrand, and Chaouki (2008) attempted to combine the DEM method with Markov chain theory. They computed the transition probability matrix directly using results obtained from a discrete element model. This work shows that, if accurate measurements of the state of the system are available, the associated Markov operator leads to a good estimate of the particle dynamics in the mixing system.

The general strategy of applying the theory of Markov chains to modeling different processes in powder technology was described by Berthiaux, Mizonov, and Zhukov (2005). It was demonstrated by
Mizonov, Berthiaux, Arlabosse, and Djerroud (2008) that the theory can be successfully used to model heat and mass transfer between stochastically moving particulate and gas flows. The results presented below for the modeling of the kinetics of ternary mixture formation are mainly based on the approach described by Mizonov, Berthiaux, and Gatumel (2016), who modeled and optimized the mixing of two dissimilar components of particulate solids.

\section{Theory}

Suppose that it is necessary to mix three dissimilar components of particulate solids, for instance, in a vibration vessel. According to the strategy of Markov chain modeling, the total height of the mixture inside the vessel $H$ is divided into $m$ perfectly mixed cells of height $\Delta x=H / m$ that can exchange components after agitation. The transition of a component from a cell can occur because of pure stochastic (symmetrical) migration of particles, characterized by the transition probability $d$, and because of segregation, characterized by the transition probability $v$. The latter can be directed downward or upward depending on the component properties and their environment. The process is observed at discrete moments of time $t_{k}=(k-1) \Delta t$, where $\Delta t$ is the time step, or transition duration, and $k$ is the transition number, which can be interpreted as the discrete analog of time. The transition probabilities can then be calculated as follows: $d=D \Delta t / \Delta x^{2}, v=V \Delta t / \Delta x$, where $D$ is the dispersion coefficient and $V$ is the dimensional velocity of segregation. For the sake of determinacy, let us suppose that the only difference between the components is their size, and assign index numbers of $1-3$ to the fine, middle, and coarse fractions, respectively.

At any moment of time $t_{k}$, the distribution of the volume content of the fractions over the cells of the chain is presented by the state column vectors $\mathbf{S}_{1}^{k}, \mathbf{S}_{2}^{k}$, and $\mathbf{S}_{3}^{k}$ of size $m \times 1$ containing elements $S_{1 j}^{k}, S_{2 j}^{k}$, and $S_{3 j}^{k}$, where $j=1, \ldots, m$ is counted from the top of the mixture.

Let us now assume that the total volume of the fractions inside each cell $S_{\max }$ remains constant with time, regardless of the composition of each fraction inside the cell. Thus, if a cell loses some of its matter during a time transition, this loss must be immediately compensated by inflows from neighboring cells. Let us assume that the value of $S_{\max }$ is equal to the conditional unit. This condition gives the following constraint

$$
S_{1 j}^{k}+S_{2 j}^{k}+S_{3 j}^{k}=1, \quad j=1, \ldots, m
$$

The state vectors $\boldsymbol{S}_{1}^{k}, \mathbf{S}_{2}^{k}$, and $\boldsymbol{S}_{3}^{k}$ vary with time, i.e., from one transition to another. Their evolution can be described by the recurrent matrix equations

$\mathbf{S}_{1}^{k+1}=\mathbf{P}_{1}^{k}\left(\mathbf{S}_{1}^{k}, \mathbf{S}_{2}^{k}, \mathbf{S}_{3}^{k}\right) \mathbf{S}_{1}^{k}$,

$\mathbf{S}_{2}^{k+1}=\mathbf{P}_{2}^{k}\left(\mathbf{S}_{1}^{k}, \mathbf{S}_{2}^{k}, \mathbf{S}_{3}^{k}\right) \mathbf{S}_{2}^{k}$,

$\mathbf{S}_{3}^{k+1}=\mathbf{P}_{3}^{k}\left(\mathbf{S}_{1}^{k}, \mathbf{S}_{2}^{k}, \mathbf{S}_{3}^{k}\right) \mathbf{S}_{3}^{k}$,

where $\mathbf{P}_{1}, \mathbf{P}_{2}$, and $\mathbf{P}_{3}$ are matrices of the transition probabilities that control the process. It is emphasized that the matrices vary from one time transition to another and depend on the current state of the mixture. Each matrix is a tridiagonal matrix of size $m \times m$. In the general case, they have the following form

$\mathbf{P}=\left[\begin{array}{lllll}P_{11} & P_{12} & 0 & 0 & \ldots \\ P_{21} & P_{22} & P_{23} & 0 & \ldots \\ 0 & P_{32} & P_{33} & P_{34} & \ldots \\ 0 & 0 & P_{43} & P_{44} & \ldots \\ \ldots & \ldots & \ldots & \ldots & \ldots\end{array}\right]$, 


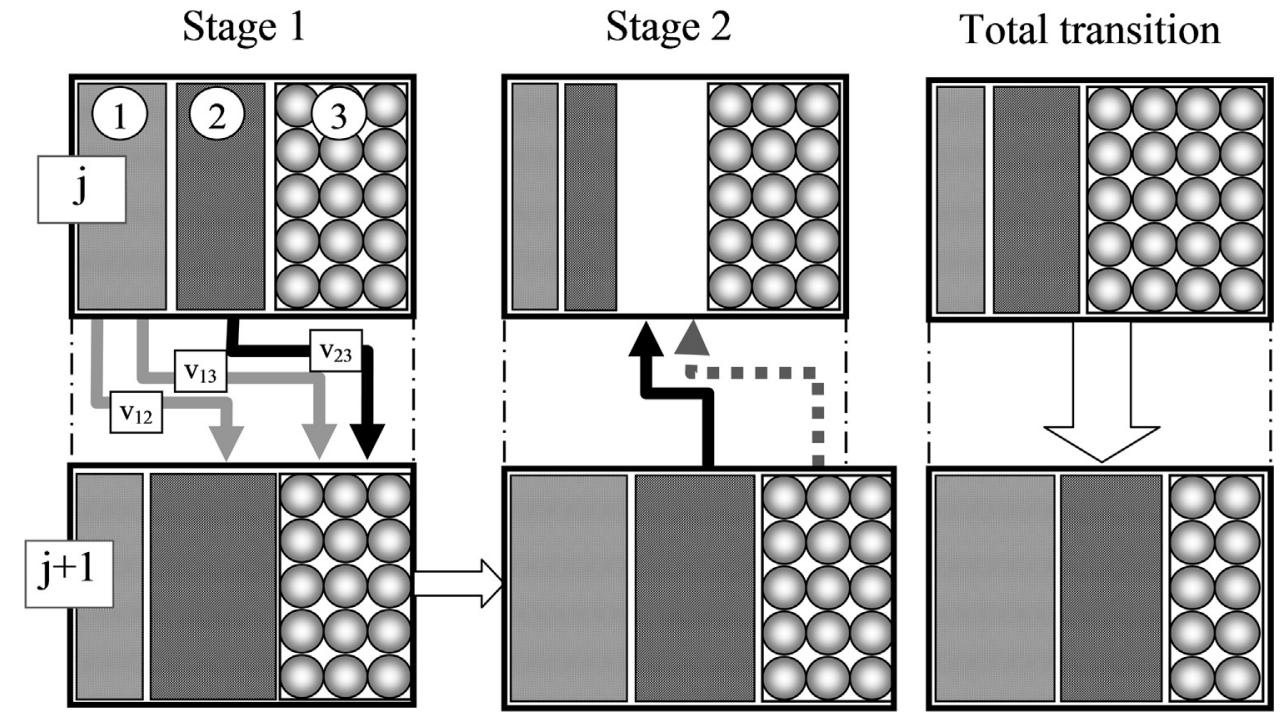

Fig. 1. Schematic representation of component migration between two neighboring cells as a result of segregation during a single transition.

where $P_{j+1, j}$ is the probability of a downward transition during $\Delta t$ (i.e., those particles that leave cell $j$ and transit to cell $j+1$ ), $P_{j-1, j}$ is the probability of upward transition during $\Delta t$ (i.e., those particles that leave cell $j$ and transit to cell $j-1)$, and $P_{j, j}$ is the probability of remaining within cell $j$ during $\Delta t$. It is obvious that $P_{j, j}$ is equal to one minus all other elements in the $j$-th column.

If downward segregation occurs, $P_{j, j+1}>P_{j-1, j}$. As mentioned above, it is convenient to separate the transitions caused by the symmetrical factor $d$ that is determined by pure stochastic mixing from the transitions caused by the non-symmetrical factor $v$ that is determined by segregation, i.e., $P_{j, j+1}=d+v$ and $P_{j-1, j}=d$. Thus, an adequate description of $v$ becomes the key point of the modeling.

The difficulty is that the fine fraction almost never segregates into the pure coarser fraction-it is only found in very small concentrations, or at the very beginning of the mixing process, when the fractions are completely separated. Mizonov et al. (2016) proposed the following description for the segregation rate based on the assumption that the fine fraction cannot segregate inside itself, that is,

$v_{j}^{k}=v_{0}\left(1-\frac{S_{j+1}^{k}}{S_{\max }}\right)=v_{0}\left(1-S_{j+1}^{k}\right)$,

where $v_{0}$ is the segregation rate of the fine fraction into the pure coarser fraction. In fact, this assumption means that the segregation only propagates into the part of the lower cell that is not occupied by the fine fraction. Substituting Eq. (6) into the cell model of binary mixture formation allows some of the physical contradictions that appear in models with constant $v_{j}^{k}$ to be excluded. Thus, the objective of the present paper is to generalize this approach to the case of a ternary mixture.

The physical sense of the proposed model and its basic assumptions are illustrated in Fig. 1. Despite the fact that the model deals with perfectly mixed cells, it is convenient to present the fraction content as vertical boxes within the cells.

The evolution of the mixture over some transition time is separated into two virtual stages that occur simultaneously. The first is downward segregation combined with pure stochastic mixing. Consider $v_{12}$ and $v_{13}$ as the rate of segregation of the fine fraction into the pure middle fraction and into the pure coarse fraction, respectively, and $v_{23}$ as the rate of segregation of the middle fraction into the pure coarse fraction. It is assumed that the segregation of the fine fraction from cell $j$ into cell $j+1$ occurs only into the volume $\left(1-S_{1, j+1}^{k}\right)$ that is free from the fine fraction and is occupied by the middle and coarse fractions with relative contents of $S_{2, j+1}^{k} /\left(S_{2, j+1}^{k}+S_{3, j+1}^{k}\right)$ and $S_{3, j+1}^{k} /\left(S_{2, j+1}^{k}+S_{3, j+1}^{k}\right)$, respectively. It is also assumed that the fine fraction transits into cell $j+1$ proportionally to these relative contents with segregation rates $v_{12}$ and $v_{13}$. This interpretation of Stage 1 of the process elicits the following formulae for the elements of the transition probability matrices $\mathbf{P}_{1}$, $\mathbf{P}_{2}$, and $\mathbf{P}_{3}$ in Eqs. (2)-(4).

Matrix $\mathrm{P}_{1}$ :

$$
P_{1, j+1, j}^{k}=d+\left(1-S_{1, j+1}^{k}\right) \frac{v_{12} S_{2, j+1}^{k}+v_{13} S_{3, j+1}^{k}}{S_{2, j+1}^{k}+S_{3, j+1}^{k}}
$$

$P_{1, j, j+1}^{k}=d$

Matrix $\mathrm{P}_{2}$ :

$P_{2, j+1, j}^{k}=d+v_{23}\left(1-S_{1, j+1}^{k}-S_{2, j+1}^{k}\right)$

$P_{2, j, j+1}^{k}=d$

Matrix $\mathrm{P}_{3}$ :

$P_{3, j, j+1}^{k}=P_{3, j+1, j}^{k}=d$

Here $j=1, \ldots, m-1$ and the probability of pure stochastic (symmetrical) transitions $d$ is assumed to be identical for all fractions. The diagonal elements of each matrix can be found from the condition of the element normalization in each column

$P_{j, j}^{k}=1-P_{j+1, j}^{k}-P_{j-1, j}^{k}$

If the transition probabilities are known, the evolution of the mixture after the first virtual stage can be calculated using Eqs. (2)-(4). This state is shown in the middle column of Fig. 1. Cell $j+1$ is overfilled with the mixture, and cell $j$ has a void volume, i.e., the condition given by Eq. (1) is not satisfied.

The second virtual stage consists of upward segregation, which can be interpreted as the void volume in cell $j$ being filled by the overflowing fractions from cell $j+1$. It is assumed that the void vol- 
ume is filled by these fractions in proportion to their content in cell $j+1$. This process can be described by the following formulae:

$\Delta S_{j}^{k+1}=1-S_{1, j}^{k+1}-S_{2, j}^{k+1}-S_{3, j}^{k+1}$,

$S_{2, j}^{k+1}:=S_{2, j}^{k+1}+\Delta S_{j}^{k+1} \frac{S_{2, j+1}^{k+1}}{S_{2, j+1}^{k+1}+S_{3, j+1}^{k+1}}$

$S_{3, j}^{k+1}:=S_{3, j}^{k+1}+\Delta S_{j}^{k+1} \frac{S_{3, j+1}^{k+1}}{S_{2, j+1}^{k+1}+S_{3, j+1}^{k+1}}$,

$S_{2, j+1}^{k+1}:=S_{2, j+1}^{k+1}-\Delta S_{j}^{k+1} \frac{S_{2, j+1}^{k+1}}{S_{2, j+1}^{k+1}+S_{3, j+1}^{k+1}}$,

$S_{3, j+1}^{k+1}:=S_{3, j+1}^{k+1}-\Delta S_{j}^{k+1} \frac{S_{3, j+1}^{k+1}}{S_{2, j+1}^{k+1}+S_{3, j+1}^{k+1}}$,

$S_{3, m}^{k+1}:=1-S_{1, m}^{k+1}-S_{2, m}^{k+1}$,

where $S_{j}^{k+1}$ is the void space in the $j$-th cell after the first stage of the $k$-th transition (The assignment operator := is used here because all the transformations occur during one time transition.).

Upon the completion of the second stage as described by Eqs. (13)-(18), the condition given by Eq. (1) is automatically satisfied.

Thus, the model given by the above equations describes the evolution of the ternary mixture state, i.e., the evolution of the distribution of its components. These equations show that a component of the mixture is solely identified by its segregation rate; the size fraction, density fraction, or fraction of another property that can influence the segregation makes no actual difference.

Some results from numerical experiments with the proposed model are presented in Figs. 2-4. The calculations were performed with $m=7$ and $v_{12}=0.2, v_{13}=0.3, v_{23}=0.1$, and $d=0.2$. The initial distribution of the fractions can be represented by the vectors $\mathbf{S}_{1}^{0}=[1$

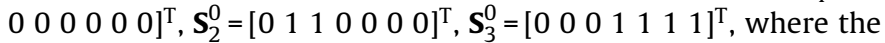
index $\mathrm{T}$ denotes the transpose of a vector.

Fig. 2 shows the evolution of the content of each component during the mixing process. The fine fraction experiences only downward segregation, and the coarse fraction experiences only upward segregation. The middle fraction experiences both upward and downward segregation, causing its distribution to be more stable and homogeneous. It follows from the physics of the process that, with no pure stochastic mixing $(d=0)$, the initial distribution should asymptotically be inverted by the segregation. Calculations show that the model does indeed give such an inverse distribution, which validates the proposed approach.

The component distribution in the mixture at different moments of time is shown in Fig. 3. The upper-left graph shows the initial distribution of the components, and the upper-right one shows the distribution in the perfectly mixed state. The lower-left graph is the component distribution after $k$ time transitions, and the lower-right graph is the asymptotical distribution. It can be seen that the non-homogeneity of the mixture first decreases, and then, after a certain amount of time, begins to increase. If $d=0$, the mixture asymptotically tends to the initial state.

The non-homogeneity of the mixture can be estimated by the standard deviation of the component distribution from the ideal mixture, $\sigma$. This standard deviation can be calculated for the whole mixture or for individual components. Fig. 4 illustrates the variation of $\sigma$ with time. It can be seen that the minimum non-homogeneity occurs at different moments of time for the individual components and for the whole mixture. The component distribution corresponding to the optimum $k=20$ is shown in Fig. 3.

Note that if identical values of $v$ are assigned to components 1 and 2 , the model for the ternary mixture transits to the model for a
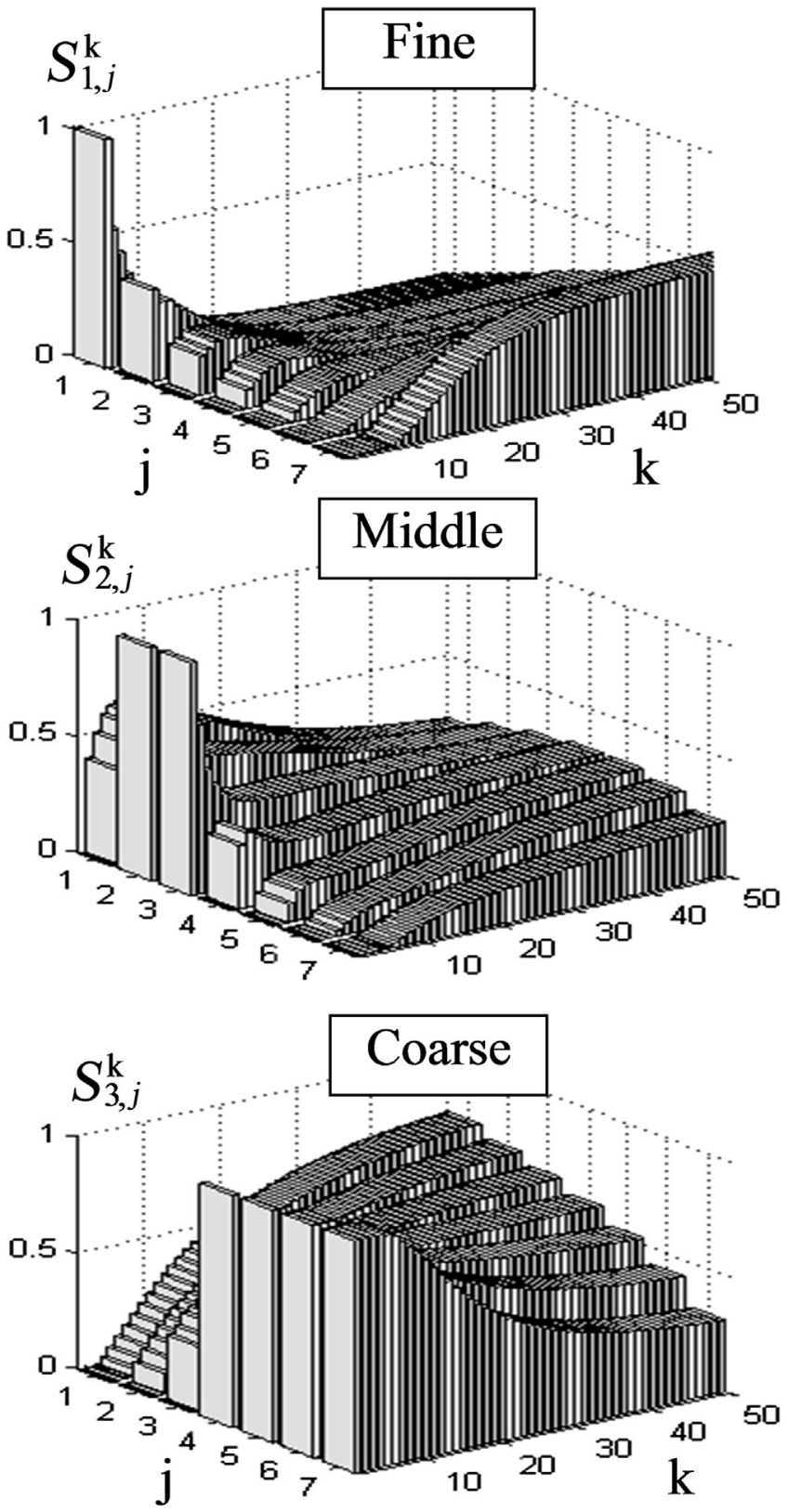

Fig. 2. Evolution of content distribution of each fraction during the mixing process.

binary mixture. The results obtained under this assumption completely coincide with the results obtained with the binary mixture model described by Mizonov et al. (2016).

The computation time for calculating the kinetics of mixing under any given conditions does not exceed $2 \mathrm{~s}$.

\section{Experimental}

An experimental validation of the model was carried out using a custom vibration stand that generates vertical vibrations of controlled amplitude and frequency. Glass beads with diameters of 2 , 4 , and $6 \mathrm{~mm}$ (with a different color for each size) were used to simulate the components to be mixed. The mixing vessel was a box with a transparent front wall of size $100 \times 150 \mathrm{~mm}$. The gap between the front and back walls was $20 \mathrm{~mm}$. A photo of the experimental set-up is shown in Fig. 5.

Using the ruler on the front wall of the box, the components were arranged in layers. After the experiment had started, the mix- 

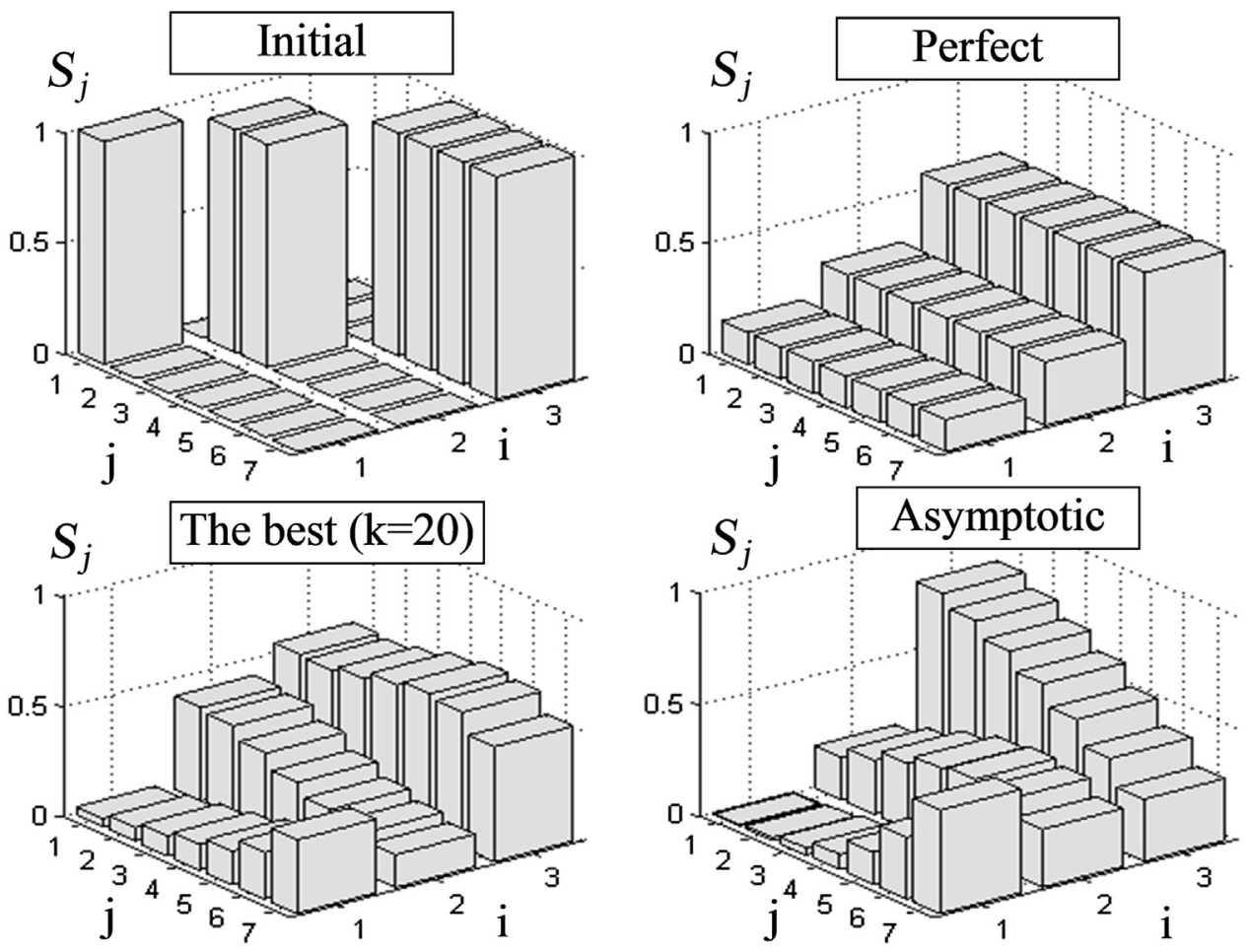

Fig. 3. State of the mixture at different moments of time.

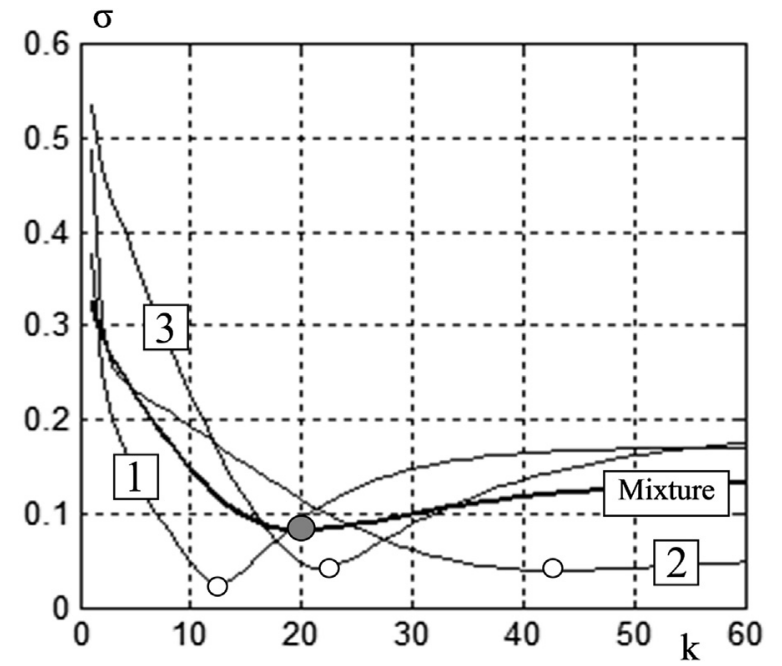

Fig. 4. Variation of $\sigma$ with time for individual components and the whole mixture.

ture was photographed every $30 \mathrm{~s}$. These pictures were analyzed using special image analysis software that allowed the content distribution of each fraction to be determined. Each experiment was repeated at least five times to obtain reproducible results, and then the average content of the fractions was calculated.

To operate the model, it was necessary to determine the downward segregation velocity of the fractions into the pure coarse fraction. This was done as follows. The thin layer of the tested fraction was placed on top of the coarser fraction, and the mixing process was launched. By analyzing the pictures, the time at which $50 \%$ of the tested fraction had migrated to the bottom cell was determined. The velocity of segregation was calculated as the distance between the coarse fraction in the top and bottom cells divided by this time period. Note that such experiments are labor

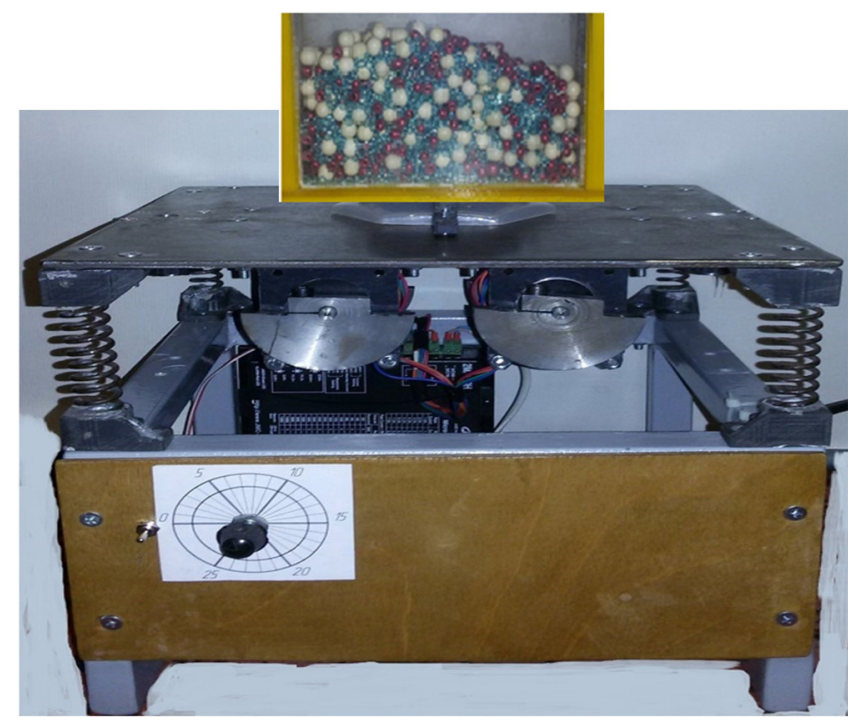

Fig. 5. Photo of the experimental set-up.

and time consuming, and it would be far more convenient to use the DEM simulation.

\section{Results and discussion}

The average segregation velocities found for the glass bead fractions according to the approach described in the previous section were broadly similar: $V_{12}=2.1 \mathrm{~cm} / \mathrm{min}, V_{13}=2.95 \mathrm{~cm} / \mathrm{min}$, and $V_{23}=1.1 \mathrm{~cm} / \mathrm{min}$. At the accepted transition duration $\Delta t=0.1 \mathrm{~min}$ and a cell height of $\Delta x=1 \mathrm{~cm}$, the corresponding segregation rates were $0.21,0.295$, and 0.11 , respectively $(v=V \Delta t / \Delta x)$. The rate of pure stochastic mixing $d$ was adjusted to minimize the sum 

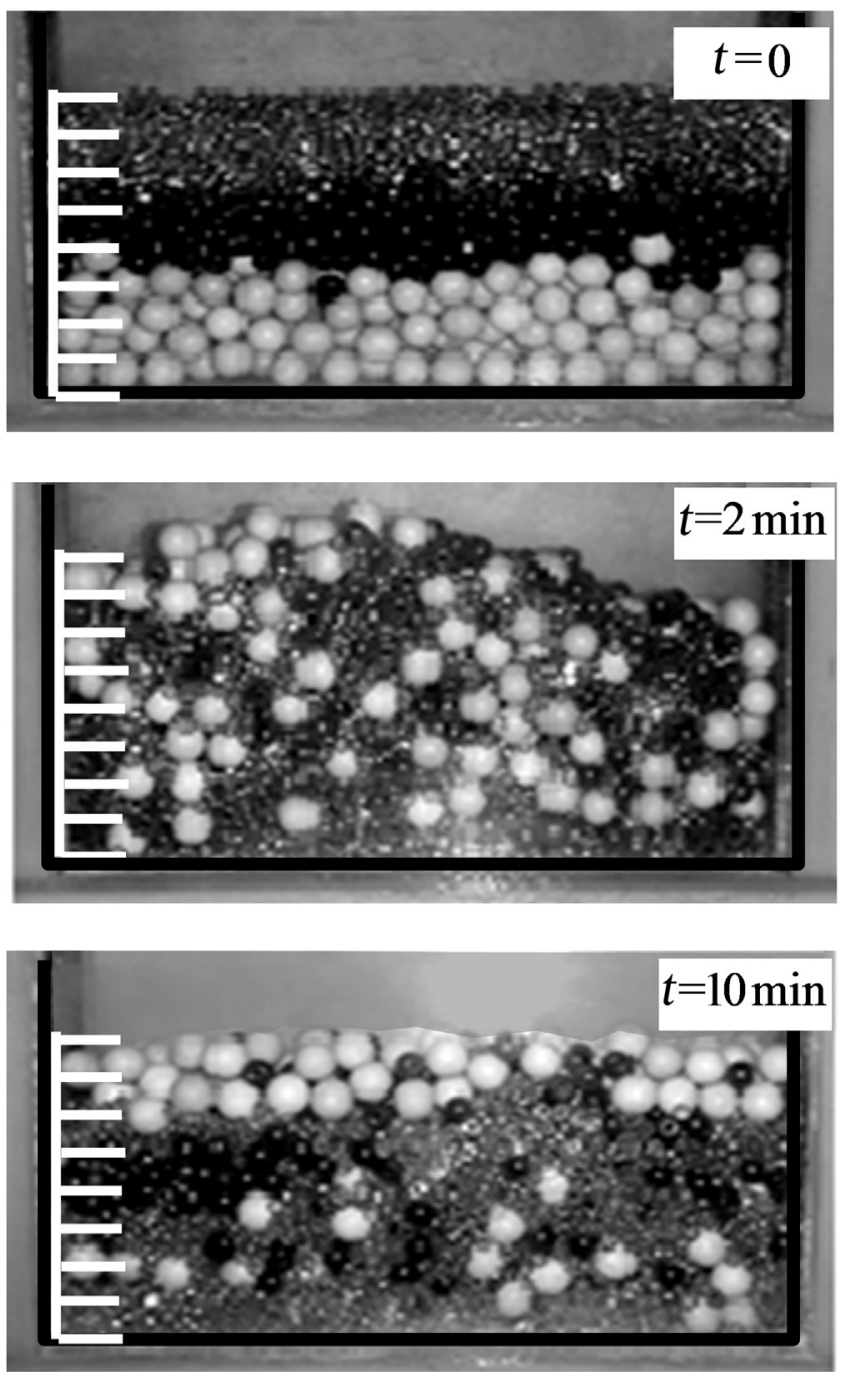

Fig. 6. Pictures of the mixture state at different moments of time.

of squared deviations of the experimental data from the modelcalculated data over the entire range of the experiment (the least-squares method).

Fig. 6 shows the mixture state at different points in time. The upper picture shows the initial state of the mixture, the middle picture shows the intermediate state after $2 \mathrm{~min}$, when the mixture has reached the best quality (minimum $\sigma$ ), and the bottom picture shows the state after $10 \mathrm{~min}$, when the distribution has practically stopped changing.

A comparison of the model-calculated and experimental data for the fraction content distributions is shown in Fig. 7. It is clear that there is a reasonable correspondence between these data.

Despite the fact that the mixture reaches its maximum quality after 2 min of mixing, the distribution of the individual components at this moment is actually very far from homogeneous. If the homogeneity of the distribution of one particular component is more important than that of the others, it is possible to determine the optimum mixing time for this specific component, or the mixing time that yields an acceptable compromise in terms of mixing quality with the other components (see, for instance, Fig. 4). Nevertheless, reaching a good mixing quality for multi-component mixtures is a complex technological problem.

It is important to remember that, according to the basic model assumptions, the phenomenon of segregation is fully determined by the downward segregation rate of a component into another pure component, regardless of whether this is due to differences in size, density, or shape. In any case, the experimental values of $V_{j}$ determined here allow the model to run and the mixing kinetics to be described.

\section{Conclusions and perspectives}

A Markov chain model of the kinetics of ternary mixture formation has been proposed. This allowed the evolution of a ternary mixture state to be calculated and the characteristics of its nonhomogeneity to be identified. It was shown that the optimum mixing time, when the mixture reaches its maximum homogeneity, does not coincide with the optimum mixing time for the individual components. Although some of the assumptions made in building the model can be disputed, the computational results are plausible because they do not contradict the physical essence of the process, yield correct asymptotic distributions, and transit to the cell model for binary mixtures after simplification. An experimental investigation with a ternary mixture of glass beads in a vertically vibrating vessel was used to validate the model, and a reasonable correlation between the experimental and calculated data was observed. The model has a very small computation time, but the experimental determination of the segregation velocities is a labor and timeconsuming process, and could be effectively replaced by a DEM simulation. Some possible avenues for model development are its 

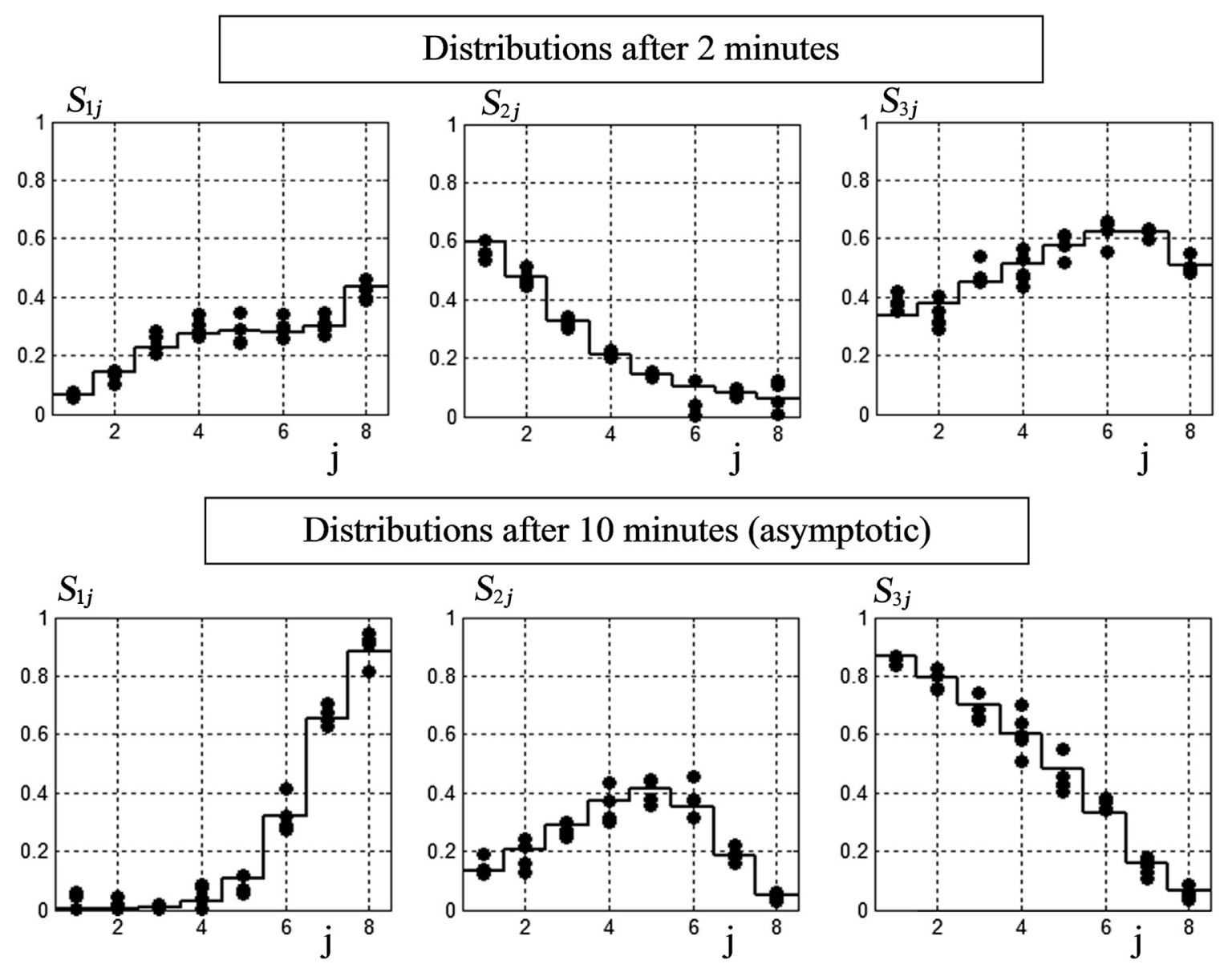

Fig. 7. Calculated (solid lines) and experimental (black circles) fraction distributions at different moments of time.

generalization to the case of an arbitrary number of dissimilar particulate solids and its use in improving the mixing quality.

\section{Acknowledgment}

This work is supported by the Russian Foundation for Basic Research (Project 15-08-01684).

\section{References}

Berthiaux, H., Mizonov, V., \& Zhukov, V. (2005). Application of the theory of Markov chains to model different processes in particle technology. Powder Technology, $157,128-137$

Bridgwater, J. (2010). Mixing of particles and powders: Where next? Particuology, 8 , 563-567.

Bridgwater, J. (2012). Mixing of powders and granular materials by mechanical means-A perspective. Particuology, 10, 397-427.

Danckwerts, P. V. (1953). Continuous flow systems: Distribution of residence times. Chemical Engineering Science, 2(1), 1-13.

Doucet, J., Hudon, N., Bertrand, F., \& Chaouki, J. (2008). Modeling of the mixing of monodisperse particles using a stationary DEM-based Markov process. Computers and Chemical Engineering, 32, 1334-1341.

Fan, L. T., Lai, F. S., Akao, Y., Shinoda, K., \& Yoshizawa, E. (1978). Numerical and experimental simulation studies on the mixing of particulate solids and the synthesis of a mixing system. Computers and Chemical Engineering, 2, 19-32.
Iddir, H., Arastoopour, H., \& Hrenya, C. M. (2005). Analysis of binary and ternary granular mixture behavior using the kinetic theory approach. Powder Technology, 151, $117-125$.

Jha, A. K., \& Puri, V. M. (2010). Percolation segregation of multi-size and multicomponent particulate materials. Powder Technology, 197, 274-282.

Jha, A. K., Gill, J. S., \& Puri, V. M. (2008). Percolation segregation in binary size mixtures of spherical and angular-shaped particles of different densities. Particulate Science and Technology, 26, 482-493.

Mizonov, V., Berthiaux, H., Arlabosse, P., \& Djerroud, D. (2008). Application of the theory of Markov chains to model heat and mass transfer between stochastically moving particulate and gas flows. Granular Matter, 10(4), 335-340.

Mizonov, V., Berthiaux, H., \& Gatumel, C. (2016). Theoretical search for solutions to minimize negative influence of segregation in mixing of particulate solids. Particuology, 25, 36-41.

Sommer, K. (1996). Mixing of particulate solids. KONA Powder and Particle Journal, $14,73-78$.

Tang, P., \& Puri, V. M. (2007). Segregation quantification of two-component particulate mixtures: Effect of particle size, density, shape, and surface texture. Particulate Science and Technology, 25, 571-588.

Wang, R. H., \& Fan, L. T. (1976). Axial mixing of grains in a motionless Sulzer (Koch) mixer. Industrial \&' Engineering Chemistry Process Design and Development, 15(3), 381-388.

Wang, R. H., \& Fan, L. T. (1977). Stochastic modeling of segregation in a motionless mixer. Chemical Engineering Science, 32, 695-701. 